\title{
Word Sense Disambiguation of Adjectives Using Probabilistic Networks
}

\author{
Gerald Chao and Michael G. Dyer \\ Computer Science Department, \\ University of California, Los Angeles \\ Los Angcles, California 90095 \\ gerald@cs.ucla.edu, dyer@cs.ucla.edu
}

\begin{abstract}
In this paper, word sense disambiguation (WSD) accuracy achicvable by a probabilistic classifier, 11sing very minimal training sets, is investigated. We mado the assumption that there are no tagged corpora available and identified what information, needed by an accurate WSD system, can and cannot be automatically obtained. The lesson learned can then be used to focus on what knowledge needs manual annotation. Our system, named Bayesian Hielarchical Disambiguator (BHD), uses the Internet, arguably the largest corpus in existence, to address the sparse data problem, and uses WordNet's hicrarchy for scmantic contextual features. In addition, Bayesian networks are automatically constructed to represent knowledge learned from training sets by modeling the selectional preference of adjectives. These networks are then applied to disambiguation by performing inferences on unseen adjective-noun pairs. We demonstrate that this system is able to clisambiguate adjectives in umestricted text at good initial accuracy rates without the need for tagged corpora. The learning and extensibility aspects of the model are also discussed, showing how tagged corpora and additional context can be incorporated easily to improve accuracy, and how this technique can be used to disambiguate other types of word pairs, such as verb-noun and adverb-verb pairs.
\end{abstract}

\section{Introduction}

Word sense disambiguation (WSD) remains an open problem in Natural Language Processing (NLP). Being able to identify the correct sense of an ambiguous word is important for many NLP tasks, such as machine translation, information retrieval, and discourse analysis. The WSD problem is exacerbated by the large number of senses of commonly used words and by the difficulty in determining relevant contextual features most suitable to the task. The absence of semantically tagged corpora makes probabilistic techniques, shown to be very effective by speech recognition and syntactic tagging rosearch, difficult to employ due to the sparse data problem.

Early NLP systems limited their domain and re- quired manual knowledge engineering. More recent works take advantage of machine readable dictionaries such as WordNet (Miller, 1990) and Roget's Online Thesaurus. Statistical techniques, both supervised lcarning from tagged corpora (Yarowsky, 1992), (Ng and Lce, 1996), and unsupervised learning (Yarowsky, 1995), (Resnik, 1997), have been investigated. There are also hybrid models that incorporate both statistical and symbolic knowledge (Wicbe et al., 1998), (Agirre and Rigau, 1996).

Supervised models have shown promising results, but the lack of sense tagged corpora often requires the need for laboriously tagging training sets manually. Depending on the technique, unsupervised models can result in ill-defined senses. Many have not been evaluated with large vocabularies or full sets of senses. Hybrid models, using various heuristics, have demonstratied good accuracy but are diffcult to compare due to variations in the ovaluation procedures, as discussed in Resnik and Yarowsky (1.997).

In our Bayesian Hicrarchical Disambignator (BHD) model, we atitempt to address some of the main issues faced by today's WSD systems, namely: 1.) the sparse data problem; 2) the selection of a feature set that can be trained upon easily without sacrificing accuracy; and 3) the scalability of the system to disambiguate unestricted text. The first two problems can be attributed to the lack of tagged corpora, while the third results from the need for hand-annotated text as a method of circumventing the first two problems. We will address the first two issues by identifying contexts in which knowledge can be obtained automatically, as opposed to those that require minimal manual tagging. The offectiveness of the BHD model is then tested on unrestricted text, thus addressing the third issue.

\section{Problem Formulation}

WSD can be described as a classification task, where the $i^{t h}$ sense $(W \# i)$ of a word $(W)$ is classified as the correct tag, given a word and usually some surrounding context. For example, to disambiguate the adjective "great" in the sentence "The 
great hurricane devastated the region", a WSD system should disambiguate "great" as lerge in size rather than the good or excellent moaning. Using probability notations, this procedure can be stated as $\max _{i}(\operatorname{Pr}($ great\#i | "groat", "tho", "hurricanc", "devastated", "the", "rogion")). "That is, given the word "great" and its context, classify the sense great $i$ with the highest probability as the correct one. However, a large context, such as the whole sentence, is rarely used, due to the difliculty in estimating the probability of this paticular set of words occurring. Therefore, the context is usually narrowed, such as $n$ number of surrounding words. Additionally, surrotnding syntactic foatures and semantic knowledge are sometimes used. The difficulty is in choosing the right context, or the set of features, that will optinize tho classification. A larger context improves the classification acenaray at the expense of increasing the number of paraneters (typically learned fiom large training datia).

Ln our BHD model, a minimal context composed of only the adjective, nom and the nom sem's semantic featmes obtained from WordNet is used. Using the above example, only "great", "lumricane" and hurricane's features encoded in WordNet's hicrarchy, as in lunricane. ISA cyclone JSA windstorm ISA violent storm..., are used as context. Therofore, the classification perforned by BHD can be written as $\max _{i}(\operatorname{Pr}($ great fti | "groat", "lnuricanc", cyclone, windstorm ...)), os nore gencrially, maxi $($ Pro(adj\#i|adj, noun, $<N$ N's $>)$ ), whore $\left\langle N F_{s}\right\rangle$ donotes the nom foatures. 13y using the Bayesian inversion formula, this equation becomes

$\max _{i}\left(\frac{\operatorname{Pr}(a d j, n o u n,\langle N F s\rangle \mid \operatorname{adj} \# i) \times \operatorname{Pr}(\operatorname{adj} \# i)}{\operatorname{Pr}\left(a d j, n o u n,\left\langle N F^{\prime} s\right\rangle\right)}\right)$.

This context is chosen because it does not need an annotated training set, and these semantic features are used to build a belief about the nomms an adjective sense typically modifies, i.e., the selectional preferences of adjectives. For example, having learned about hurricane, the system can infer the most probable disambiguation of "great typhoon", "great tornado", or more distal concepts such as earthquakes and floods.

\section{Establishing the Parameters}

As shown in equation 1, BHJ) requires two parameters: 1) the likelihood term $\operatorname{Pr}(\operatorname{adj}$, noun, $\langle N F s\rangle \mid a d j \# i)$ and 2) the prior term $\operatorname{Pr}(a d j \neq i)$. The prior term represents the knowledge of how frequently a sense of an adjective is used without any contextual information. For example, if great\#2 (sense: good, exeellent) is used frequently while great 1 is less commonly used, then Pr(great\#2) would be larger than
Pr(grcat\#1), in proportion to the wage of the two senses. Although WordNet orders the senses of a polysemous word according to usage, the actmal proportions are not quantified. Therefore, to compute the priors, one can itcrate over all English nouns and sum the instiances of great versus great ft2-noun pairs. But since wo assume that no training set exists (the worst possible case of the sparse (late problem), these comnts need to be estimated from indirect sources.

\subsection{The Sparse Data Problem}

Tho technique used to address data sparsity, as first proposed by Milalcea and Moldovan (1998), treats the Internet as a corpus to automatically disambiguate word pairs. Using the previous example, to disambiguate the adjective in "great hurricane", two symonym lists of ("great, large, big") and ("great, neat, good") aro rotrieved from WordNot. (Some symonyms and other semses are onitied here for brevity.) Two quories, ("great humricane" or "large hutricane" or "big hurricanc") and ("great hurricane" or "neat hurricane" or "good hurricane"), are issued to Altavista, which reports that 1100 and 914 pages contain these terms, respectively. The query with the higher comb $(\# 1)$ is classified as the correct sense. For further details, ploase refer to Milalcea and Moldovan (1998).

In our nodel, the counts from Altavista are incorporated ats paranceter estimations within our probatbilistic: framework. In addition to disanbloguating the adjectives, we also need to estimate the usage of the acljectivefti-nom pair. For simplicity, the counts fiom Altavista are assigned wholesale to the disambiguated adjective sonse, e.g., the usage of groat\#-hurricane is 1100 times and great\#2humricano is zero times. This is a great simplifiation since in many adjective-noun pairs multiple meanings are likely. For instance, in "groat steak", botll scnse of "great" (large steak vs. tasty steak) are equally likely. However, given no other information, this simplification is used as a gross approximation of Counts(adj\#i-notm), which becomess $\operatorname{Pr}$ (adj\#i-noun) by dividing the comts by a nomalixing constant, $\sum$ Counts(adjłi-all nouns). These probabilities are then used to compute the priors, (lescribed in the next section.

Using this technique, two major problems are addressed. Not only are the adjectives antomatically disambiguated, but the number of occurrences of the word pairs is also estimated. The need for handannotated semantic corpora is thus avoided. However, the statistics gathered by this technicue are approximations, so the noise they introduce does nequire supervised training to minimize cror, as will be rlescribod. 


\subsection{Computing the Priors}

Using the methods described above, the priors can be automatically computed by iterating over all nouns and summing the counts for each adjective sense. Unfortunately, the automatic disambiguation of the adjective is not reliable cnough and results in inaccurate priors. Therefore, manual classification of assigning nouns into one of the adjective senses is needed, constituting the first of two manual tasks needed by this model. However, instead of classifying all English nouns, Altavista is again used to provide collocation data on 5,000 nouns for each adjective. The collocation frequency is then sorted and the top 100 nouns are manually classified. For example, the top 10 nouns that collocate after "great" are "deal", "site", "job", "place", "time", "way", "American", "page", "book", and "work". They are then all classificd as being modified by the great \#2 sense except for the last one, which is classified into another sense, as defined by WordNet. The prior for each sense is then computed by summing the counts from pairing the adjective with the nouns classified into that sense and dividing by the sum of all adjective-noun pairs. The top 100 collocated nouns for each adjective are used as an approximation for all adjective-noun pairs since considering all nouns would be impractical.

To validate these priors, a Naive Bayes classifier that computes

$$
\max _{i} \frac{\operatorname{Pr}(a d j, n o u n \mid a d j \# i) \times \operatorname{Pr}(\operatorname{adj} \# i)}{\operatorname{Pr}(a d j, \text { noun })}
$$

is used, with the noun as the only context. This simpler likelihood term is approximated by the same Internet counts used to establish the priors. i.e., $\approx$ Counts(adj\#i-noun) / normalizing constant. In Table 1 , the accuracy of disambiguating 135 adjectivenoun pairs from the br-a01 file of the semantically tagged corpus SemCor (Miller et al., 1993) is compared to the baseline, which was calculated by using the first WordNet sense of the adjective. As mentioned earlier, disambiguating using simply the highest count from Altavista ("Bofore Prior" in Table 1) achicved a low accuracy of $56 \%$, whereas using the sonse with the highest prior ("Prior Only") is slightly better than the bascline. This result validates the fact that the priors established hore preserve WordNet's ordering of sense usage, with the improvement that the rclative usages between senses are now quantified.

Combining both the prior and the likelihood terms did not significantly improve or degrade the accuracy. This would indicate that either the likelihood term is uniformly distributed across the $i$ senses, which is contradicted by the accuracy without the priors (second row) being significantly higher than the average number of senses per adjective of 3.98 ,

\begin{tabular}{|l|r|}
\hline & Accuracy \\
\hline \hline Before Prior & $56.3 \%$ \\
Prior Only & $77.0 \%$ \\
Combined & $77.8 \%$ \\
\hline Baseline & $75.6 \%$ \\
\hline
\end{tabular}

Table 1: Accuracy rates from using a Naive Bayes classifier to validate the priors. These results show that the priors established in this model are as accurate as the WordNet's ordering according to scnse usage (Baseline).

or, more likely that this parameter is subsumed by the priors due to the limited context. Therefore, more contextual information is needed to improve the model's performance.

\subsection{Contextual Features}

Instead of adding other types of context such as the surrounding words and syntactic features, the semantic features of the noun (as cncoded in the WordNet ISA hierarchy) is investigated for its effectiveness. These foatures are readily available and are organized into a well-defined structure. The hierarchy provides a systematic and intuitive mothod of distance measurements between feature vectors, i.e., the semantic distance between concepts. This property is very important for inferring the classification of the novel pair "great flood" into the sense that, contains hurricane as a member of its prototypical nouns. These prototypical nouns describe tho sclectional preferences of adjective senses of "great", and the semantic distance between them and a now noun measures the "semantic fit" between the concepts. The closer they are, as with "hurricane" and "flood", the higher the probability of the likelihood term, whercas distal concepts such as "hurricano" and "taste" would have a lower value.

Representing these prototypical nouns probabilistically, however; is difficult due to the exponential number of probabilities with respect to the number of features. For cxample, representing hurricane being present in a selectional preference list requires $2^{8}$ probabilitios since there are 8 features, or ISA parents, in the WordNet hicrarchy. In adclition, the sparse data problem resurfaces because each one of the $2^{8}$ probabilities has to be quantified. To address these two issues, bolief networks are used, as described in detail in the next section.

\section{Probabilistic Networks}

There are many advantages to using Bayesian networks over the traditional probabilistic models. The most notable is that the number of probabilities needed to represent the distribution can be significantly reduced by making independence assumptions between variables, with cach node condition- 


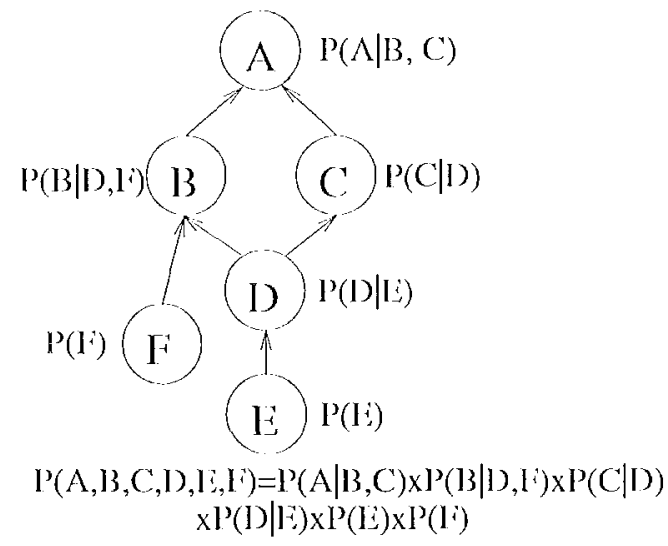

Figure 1: An example of a Bayesian notwork and the probabilitios at each node that dofine the rolationships between a node and its parents. 'I'le equation at the bottom shows how the distribution across all of the variables is computed.

ally dependent upon only its paronts (l'oarl, 1988). Figne I slows an exannlo bayesian network representing the distribution $\mathrm{P}(\Lambda, \mathrm{B}, \mathrm{C}, 1), \mathrm{T}, \mathrm{J})$. Instead of having one large table with $2^{\text {fi }}$ probabilities (with all Boolean nodes), the distribution is represented by the conditional probability tables (CP'I's) at each

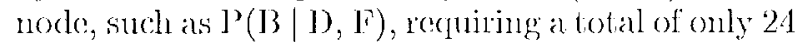
probalbilities. Not only do the savings become norer significant with larger notworks, but the sparse data problem becomes more manageable as well. The taining set no longer noeds to cover all permutattions of the feature sets, but only smatlen sulbsets dictated by the seds of valiables of the Cl'T's.

The networls shown in Figne 1 looks sinilax to any portion of the WordNet hionarchy for a roason. In BOID, bolicf networks with the same structure as the WordNet hierarehy are antomatically constructed to represent the selectional preforence of an adjective senso. Specifically, the network represonts the probalbilistic distribution over all of the prototypjeal nomes of an astjectiveffi ancl the nouns' semantic featunes, i.e., P(protonouns, < protoNl's > |adj\#i). 'L'he use of Bayesian networks for WSD has been proposed by others such as Wiebe et. al (1998), but a diflerent formulation is used in this model. The construction of the networks in BHD can be divided into three steps: defining 1) the training sets, 2) the structure, and 3) the probabilities, as described in the following sections.

\subsection{Training Sets}

The training set for each of the adjective senses is constructed by extracting the exemplary adjectivenoun pairs from the WordNet glossary. The glossary contains the example usage of the adjectives, and the nouns from them are taken as the training sots

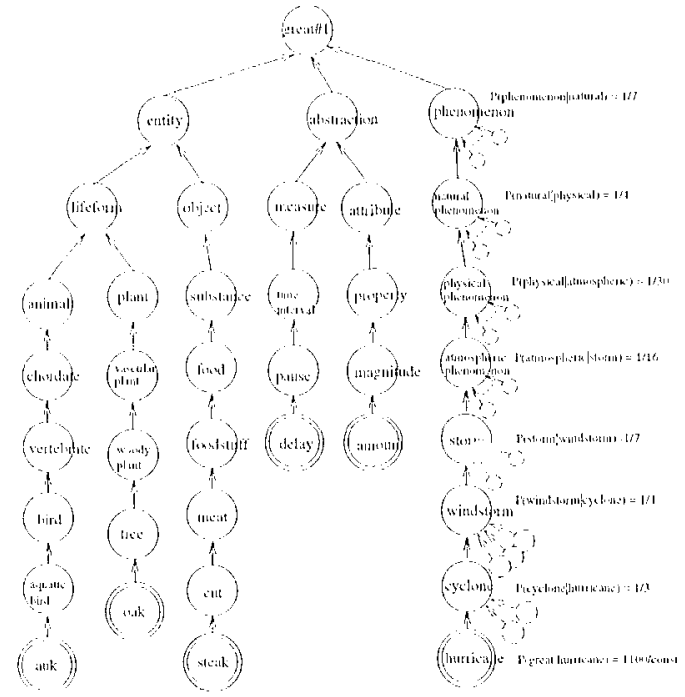

Figure 2: The stancture of the belief network that represents the solectional pueference of greatfl. 1 . The leaf nodes are the nouns within the training set, and the intermediate nodes refled the ISA hienarely. from WordNet. Tho probabilities at each node aro used to disambignate novel adjective-nom pairs.

for the adjectives. For example, the nom "ank", "oak", "steak", "dolay" and "amount" compose the training set for great 1 (sense: large in size). Note 1. lat WordNet included "stcals" in the glossary of great ff 1 , but it appears that the good or eacellent sense would bo more appopmiate. Nexertheless, the lists of exemplary nomms are systematically retriexed and not exlited.

'the sets of prototypical nouns for each adjective sense haxe to be disambignated because the semantic features cliffer between ambiguous nouns. Since these nomns camnot be antomatically disambignated with high accuracy, they have to be done manually. 'This is the second part of the mamual process needed by BHD since the WordNet glossary is not semantically tagged.

\subsection{Belief Network Structure}

The belief networks have the sane structure as the Word INet ISA hierarchy with the exception that the edges are directed from the child nodes to their parents. Illustrated in Figure 2, the BHD-constructed network represents the selectional preference of the top level node, great \#1. The leaf nodes are the evidence nodes from the training set and the intermediate nodes are the semantic features of the leaf nodes. This organization enables the belief gathered from the leaf norles to be propagated up to the top level node during inferencing, as described in a later section. I3ut first, the probability table accompanying aach node needs to be constructed. 


\subsection{Quantifying the Network}

The two parameters the belief networks require are the CPTs for each intermediate node and the priors of the leaf nodes, such as P(grcat\#1, hurricanc). The latter is estimated by the counts obtained from Altavista, as described earlier, and a shortcut is used to specify the CPTs. Normally the CPTs in a fully specified Bayesian network contain all instantiations of the child and parent values and their corresponding probabilities. For cxample, the CPT at node D in Figure 1 would have four rows: $\operatorname{Pr}(D=t \mid E=t), \operatorname{Pr}(D=t \mid E=f), \operatorname{Pr}(D=f \mid E=t)$, and $\operatorname{Pr}(\mathrm{D}=\mathrm{f} \mid \mathrm{E}=\mathrm{f})$. This is needed to perform full inferencing, where queries can be issued for any instantiation of the variables. However, since the networks in this model are used only for onc specific query, where all nodes are instantiated to be true, only the row with all variables cqual to true, e.g., $\operatorname{Pr}(D=t \mid E=t)$, has to be specified. The nature of this query will be described in more detail in the next section.

To calculate the probability that an intermediate node and all of its parents are true, one divides the number of parents present by the number of possible parents as specificd in WordNet. In Figure 2, the small dotted nodes denote the absent parents, which determine how the probabilities are specified at each node. Recall that the parents in the belief network are actually the children in the WordNet hicrarchy, so this probability can be seen as the percentage of children actually present. Intuitively, this probability is a form of assigning weights to parts of the network where more related nouns are present in the training set, similar to the concept of semantic density. The probability, in conjunction with the structure of the belief notwork, also implicitly encodes the semantic distance between concepts without necessarily penalizing concepts with decp hicrarchies. A discount is taken at each ancestral node cluring inferencing (next section) only when some of its WordNet children are absent in the network. Therefore, the semantic distance can be secn as the number of traversals up the network weighted by the number of siblings present in the tree (and not by direct edge counting).

\subsection{Querying the Network}

With the probability between nodes specified, the network becomes a representation of the selectional preference of an adjective sense, with features from the WordNet ISA hierarchy providing additional knowledge on both semantic densities and semantic distances. To disambiguate a novel adjective-noun pair such as "great flood", the great\#1 and great\#2 networks (along with 7 other great\# $i$ networks not shown here) infor the likelihood that "flood" belongs to the network by computing the probability
$\operatorname{Pr}$ (great, flood, < llood NFs>, proto nouns, < proto NFs $>\mid$ adj\#i), even though neither network has evor cncountered the noun "flood" before.

To perform these inferences, the noun and its features are temporarily inserted into the network according to the WordNet hierarchy (if not already present). The prior for this "hypothetical cvidence" is obtained the same way as the training set, i.e., by quorying Altavista, and the CPTs are updated to reflect this new addition. To calculate the probability at the top node, any Bayesian network inferencing algorithm can be used. However, a query where all nodes are instantiated to true is a special case since the probability can be computed by multiplying together all priors and the CP'T entries where all variables are true.

In Figure 3, the network for great $\# 1$ is shown with "flood" as the hypothetical evidence added on the right. The CPT of the node "natural phenomenon" is updated to reflect the newly added evidence. The propagation of the probabilities from the leaf nodes up the network is shown and illustrates how discounts are taken at each intermediate node. Whenever more related concepts are present in the network, such as "typhoon" and "tornado", less discounts are taken and thus a higher probability will result at the root node. Conversely, one can see that with a distal concept, such as "taste" (which is in a completely different branch), the knowledge about "hurricane" will have little or no influence on disambiguating "great taste".

The calculation above can be computed in lincar time with respect to the depth of the query noun node (depth $=5$ in the case of flood \#1) and not the the number of nodes in the network. This is important for scaling the network to represent the large number of nouns needed to accurately model the selectional preferences of adjective senses. The only cost incurred is storage for a summary probability of the children at each intermediate node and time for updating these values when a new piece of evidence is added, which is also lincar with respect to the depth of the node.

Finally, the probabilities computed by the inference algorithm are combined with the priors established in the earlier section. The combined probabilities represent $P(\operatorname{adj} \# \mathbf{i} \mid \operatorname{adj}$, noun, $\langle N F s>$ ), and the one with the highest probability is classified by BHD as the most plausible sense of the adjective.

\subsection{Evaluation}

To test the accuracy of BHD, the same procedure described earlier was used. The same 135 adjectivenoun pairs from SemCor were disambiguated by BHD and compared to the bascline. Table 2 shows the accuracy results from cvaluating either the first sense of the nouns or all senses of the nouns. The results of the accuracy without the priors $\operatorname{Pr}(\operatorname{adj} \# \mathrm{i})$ in- 


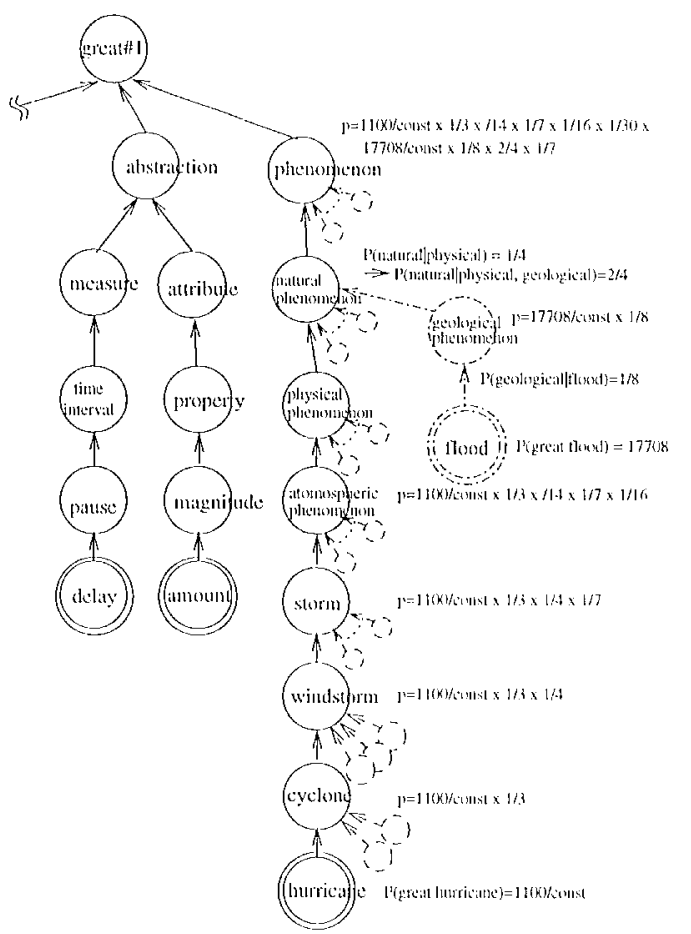

Figure 3: Query of the great\#1 bolief network to infer the probability of flood being morlified by great\#1. The left branch of the network has been omitted for clarity.

dicate the improvements provided by the likelihood term alone. The innprovement galined from the additional contextual features shows the effectiveness of the belief networks. Ifven with only 3 pototypical nomms per adjective sonse on arerage (hadly a complete description of the selectional preferences) the gain is very conconaging. With the priors factored in, BHD improved even further $(81 \%)$, significantly surpassing the baseline (75.6\%), a feat acconplished by only one other inodel that, we are aware of (Jiri Stetina and Nagao, 1998). Note that the bost accuracy was achioved by ovaluating all senses of the nouns, as expected, since the solectional preference is modeled through scmantic foatures of the glossary nouns, not just their word forms. The reason for the good accuracy from using only the first noun sonse is because $72 \%$ of them happen to be the first sense. These results are very encouraging since no tagged corpus and minimal training datia were used. Wo believe that with a bigger training set, BHD's performance will inprove even further.

\subsection{Comparison with Other Models}

To our knowledge, there are only two other systems that disambiguate acljective-noun pairs fiom untestricted text. Results from both models were evaluated against SemCor and thus a comparison is noaningful. In Table 3, each model's accuracy (as well as

\begin{tabular}{|c|c|c|c|}
\hline & Context & $\begin{array}{r}1^{s t} \text { noun } \\
\text { sense }\end{array}$ & $\begin{array}{l}\text { all now11 } \\
\text { sonses }\end{array}$ \\
\hline \multirow{2}{*}{$\begin{array}{l}\text { Without } \\
\text { Jrior }\end{array}$} & noun only & $56.3 \%$ & $53.3 \%$ \\
\hline & $+\mathrm{SP}$ & $60.0 \%$ & $60.0 \%$ \\
\hline With & nonin only & $77.8 \%$ & $77.8 \%$ \\
\hline Prior: & $+\mathrm{SP}^{3}$ & $80.0 \%$ & $81.4 \%$ \\
\hline \multicolumn{2}{|l|}{ Jatseline } & $75.6 \%$ & $75.6 \%$ \\
\hline
\end{tabular}

Table 2: Accuracy results from the selectional preference model ( $+\mathrm{SP}$ ), showing the improvements over the baseline by either considering the first noun sense or all nom senses.

\begin{tabular}{|l|c|c|}
\hline Model & Results & Baseline \\
\hline \hline BHII) & $81.4 \%$ & $75.6 \%$ \\
\hline $\begin{array}{l}\text { Mihalcea and Moldowan } \\
\text { (1999) }\end{array}$ & $79.8 \%$ & $81.8 \%$ \\
\hline Stetina of al. (1998) & $83.6 \%$ & $81.9 \%$ \\
\hline
\end{tabular}

Table 3: Comparison of adjective disambiguation ac:curay with other models.

the baseline) is provided since different adjectivenoun pairs were evaluated. Wo find the BHJ) results comparable, if not better, especially when the amount of inprovement over the baseline is comsiclered. The model by Stetina (1998) was trained on SemCor that was nerged with a full sentential parse troe, the determination of which is considered a difficult problem of its own (Collins, 1997). We beliere that by incorporating the datia from SemCor (discussed in the futme work section), the performanee of our system will surpass Stetinal's.

\section{Conclusion and Future Work}

Wo have prosented a probalilistic disambignation model that is systematic, accurate, and require mannal intervention in only in two places. 'The more time consunning of the two manual tasks is to classify the top) 100 nouns noeded for the priors. The other task, of clisambiguating prototypical nouns, is relatively simple due to the limited number of glossary nouns per sonse. However, it, would be straightforward to incorporate semantically tagged corpora, such as SemCor, to avoicl these manual tasks. The priors are the number of instances of each adjective sense divided by all of the adjectives in the corpus. The disambiguated adjective\#i-noun\#j pairs from the corpus can be used as training sets to build better representiation of solectional preferences by inserting the nom \#j node and the accompany fartmes into the belief network of adjectiveffi. The insertion is the same procelure used to add the hypothetical evidence during the inferencing stage. 'The upelated belief notworks could then bo used for disambiguation with improved accuracy. Furthermore, the performance of BIHD could also be improved by expand- 
ing the context or using statistical learning methods such as the EM algorithm (Dempster et al., 1977). Using Bayesian networks gives the model flexibility to incorporate additional contexts, such as syntactical and morphological features, without incurring exorbitant costs.

It is possible that, with an extended model that accurately disambiguates adjective-noun pairs, the sclectional preference of adjectivo senses could be automatically learned. Having an inproved knowledgo about the selectional preferences would then provide better parameters for disambiguation. The model can be seen as a bootstrapping loarning process for disambiguation, where the information gained from one part (sclectional preference) is used to improvo the other (disambiguation) and vice versa, reminiscent of the work by Riloff and Jones (1999) and Yarowsky (1995).

Lastly, the techniques used in this paper could be scaled to disambiguate not only all adjective-noun pairs, but also other word pairs, such as subjectverb, verb-object, adverb-verb, by obtaining most of the parameters from the Internet and WordNet. If the information from SemCor is also used, then the system could be automatically trained to perform disambiguation tasks on all content words within a sentence.

In this paper, we have addressed three of what we believe to be the main issues faced by current WSD systems. We demonstrated the effectiveness of the techniques used, while identifying two manual tasks that don't necessarily require a semantically tagged corpus. By establishing accurate priors and small training sets, our system achieved good initial disambiguation accuracy. The same methods could be fully automated to disambiguate all content word pairs if information from semantically tagged corpora is used. Our goal is to create a system that can disambiguate all content, words to an accuracy level sufficicnt for automatic tagging with human validation, which could then be used to improve or facilitate new probabilistic semantic taggers accurate enough for other NLP applications.

\section{References}

Eneko Agirro and German Rigau. 1996. Word sense disambiguation using conceptual density. In Proceedings of COLING-96, Copenhagen.

Michacl Collins. 1997. Three generative, lexicalised models for statistical parsing. In Proceedings of the 35th Annual Meeting of the ACL, pages 1623, Madrid, Spain.

A.P. Dempster, N.M. Laird, and D.B. Rubir. 1977. Maximum likelihood from incomplete data via the EM algorithm. Journal of the Royal Statistical Society, 39(B):1-38.
Sadao Kurohashi Jiri Stetina and Makoto Nagao. 1998. Gencral word sense disambiguation mothod based on a full sentential context. In Proceedings of COLING-ACL Workshop on Usage of WordNet in Natural Language Processing, Montreal, Canada, July.

Rada Mihalcea and Dan Moldovan. 1998. Word sense disambiguation based on semantic density. In Proceedings of COLING-ACL Workshop on Usage of WordNet in Natural Language Processing, Montreal, Canada, July.

G. Miller, C. Leacock, and R. Tengi. 1993. A semantic concordance. In Proccedings of ARPA Human Language Technology, Princeton.

G. Miller. 1990. WordNet: An on-line lexical database. International Journal of Lexicoyraphy, $3(4)$.

Hwee Tou Ng and Hian Beng Lee. 1996. Integrating multiple knowledge sources to disambiguate word sense: An exemplar-based approach. In Proceedings of the 34th Annual Mecting of ACI, Santa Cruz, June.

Judea Poarl. 1988. Probabilistic Reasoning in Intelligent Systems: Netuorks of Plausible Inference. Morgan Kaufmann, San Matco, CA.

Philip Resnik and David Yarowsky. 1997. A perspective on word sense disambiguation methods and their evaluation. In ANLP' Workshop on Tagging Text with Lexical Semantics, Washington, D.C., June.

Philip Resnik. 1997. Selectional proforence and scnse disambiguation. In ANLP Workshop on Tagging Text with Lexical Semantics, Washington, D.C., June.

Ellon Riloff and Rosic Jones. 1999. Lcanning dictionaries for information extraction by multi-level bootstrapping. In Proceedings of AAAI-99, Orlando, Florida.

Janyce Wiebe, Tom O'Hara, and Rebecca Bruce. 1998. Constructing bayesian networks from WordNet for word-sense disambiguation: Representational and processing issues. In Proceedings of COLING-ACL Workshop on Usage of WordNet in Natural Language Processing, Montreal, Canada, July.

David Yarowsky. 1992. Word-sense disambiguation using statistical model of Roget's categorics trained on large corpora. In Proceedings of COLING-92, Nantes, France.

David Yarowsky. 1995. Unsupervised word sense disambiguation rivaling supervised methods. In Proceedings of the 33rd Annual Meeting of the $A C L$. 